\title{
Brodalumab in an Organ Transplant Recipient With Psoriasis
}

\author{
Indira Singh, MD; Angeline Uy, MD, MBA; Ari Kassardjian, BS; April W. Armstrong, MD, MPH
}

\section{PRACTICE POINTS}

- Immunocompromised patients, such as organ transplant recipients, require careful benefit-risk consideration when selecting a systemic agent for psoriasis.

- Brodalumab, an IL-17RA antagonist, was used to treat a patient with psoriasis who had undergone solid organ transplant with excellent response and good tolerability.

- Further studies are needed to evaluate the benefits and risks of using biologic treatments in patients with psoriasis who are organ transplant recipients.

Immunocompromised patients, such as organ transplant recipients, require careful benefit-risk consideration when selecting a systemic agent for psoriasis. Brodalumab is an immunomodulatory biologic that binds to and inhibits IL-17RA, thereby inhibiting the actions of IL-17A, F, E, and C. Brodalumab has a rapid onset of action, sustained efficacy, and an acceptable safety profile, all of which serve to enhance its appeal as a systemic treatment option for psoriasis in immunocompromised patients. Reports of brodalumab use for psoriasis in organ transplant recipients are limited. We report a case in which brodalumab was used to treat psoriasis in a patient who had undergone solid organ transplantation with excellent response and good tolerability.

Cutis. 2021;107:104-106.

\footnotetext{
\rceil
} he treatment landscape for psoriasis has evolved rapidly over the last decade. Biologic therapies have demonstrated robust efficacy and acceptable safety profiles among many patients with moderate to severe plaque psoriasis. However, the use of biologics among immunocompromised patients with psoriasis rarely is discussed in the literature. As new biologics for psoriasis are being developed, a critical gap exists in the literature regarding the safety and efficacy of these medications in immunocompromised patients. Per American Academy of Dermatology-National Psoriasis Foundation guidelines, caution should be exercised when using biologics in patients with immunocompromising conditions. ${ }^{1}$ In organ transplant recipients, the potential risks of combining systemic medications used for organ transplantation and biologic treatments for psoriasis are unknown. ${ }^{2}$

In the posttransplant period, the immunosuppressive regimens for transplantation likely will improve psoriasis. However, patients with organ transplant and psoriasis still experience flares that can be challenging to treat. ${ }^{3}$ Prior treatment modalities to prevent psoriasis flares in organ transplant recipients have relied largely on topical therapies, posttransplant immunosuppressive medications (eg, cyclosporine, tacrolimus, mycophenolate mofetil) that prevent graft rejection, and systemic corticosteroids. We report a case of a 50-year-old man with a recent history of liver transplantation who presented with severe plaque psoriasis and psoriatic arthritis.

\section{Case Report}

A 50-year-old man presented to the dermatology clinic with moderate to severe plaque psoriasis and psoriatic arthritis that had been present for 15 years. His plaque psoriasis covered approximately $40 \%$ of the body surface area, including the scalp, trunk, arms, and legs. In addition, he had diffuse joint pain in the hands and feet; a

From the Department of Dermatology, University of Southern California Keck School of Medicine, Los Angeles.

Drs. Singh and Uy and Mr. Kassardjian report no conflict of interest. Dr. Armstrong has served as a consultant or research investigator for AbbVie,

Bristol Myers Squibb, Dermavant Sciences, Dermira, Eli Lilly and Company, Janssen Pharmaceutica, LEO Pharma, Modernizing Medicine, Novartis,

Ortho Dermatologics, Regeneron Pharmaceuticals, Sanofi Genzyme, and UCB.

Correspondence: Indira Singh, MD, Keck School of Medicine at University of Southern California, Norris Comprehensive Cancer Center,

1441 Eastlake Ave, Topping Tower, Ste 3427, Los Angeles, CA 90033 (indira.Singh@med.usc.edu).

doi:10.12788/cutis.0167 
radiograph revealed active psoriatic arthritis involving the joints of the fingers and toes.

One year prior to presentation to our dermatology clinic, the patient underwent an an orthotopic liver transplant for history of Child-Pugh class $\mathrm{C}$ liver cirrhosis secondary to untreated hepatitis $\mathrm{C}$ virus (HCV) and alcohol use that was complicated by hepatocellular carcinoma. He acquired a high-risk donor liver that was HCV positive with HCV genotype 1a. Starting 2 months after the transplant, he underwent 12 weeks of treatment for HCV with glecaprevir-pibrentasvir. Once his HCV treatment course was completed, he achieved a sustained virologic response with an undetectable viral load. To prevent transplant rejection, he was on chronic immunosuppression with tacrolimus, a calcineurin inhibitor, and mycophenolate mofetil, an inhibitor of inosine monophosphate dehydrogenase whose action leads to decreased proliferation of T cells and B cells.

The patient's psoriasis initially was treated with triamcinolone acetonide ointment $0.1 \%$ applied twice daily to the psoriasis lesions for 1 year by another dermatologist. However, his psoriasis progressed to involve $40 \%$ of the body surface area. Following our evaluation 1 year posttransplant, the patient was started on subcutaneous brodalumab $210 \mathrm{mg}$ at weeks 0,1 , and 2, then every 2 weeks thereafter. Approximately 10 weeks after initiation of brodalumab, the patient's psoriasis was completely clear, and he was asymptomatic from psoriatic arthritis. The patient's improvement persisted at 6 months, and his liver enzymes, including alkaline phosphatase, total bilirubin, alanine transaminase, and aspartate transaminase, continued to be within reference range. To date, there has been no evidence of posttransplant complications such as graft-vs-host disease, serious infections, or skin cancers.

\section{Comment}

Increased Risk for Infection and Malignancies in Transplant Patients-Transplant patients are on immunosuppressive regimens that increase their risk for infection and malignancies. For example, high doses of immunosuppresants predispose these patients to reactivation of viral infections, including BK and JC viruses. ${ }^{4}$ In addition, the incidence of squamous cell carcinoma is 65- to 250-fold higher in transplant patients compared to the general population. ${ }^{5}$ The risk for Merkel cell carcinoma is increased after solid organ transplantation compared to the general population. ${ }^{6}$ Importantly, transplant patients have a higher mortality from skin cancers than other types of cancers, including breast and colon cancer. ${ }^{7}$

Psoriasis in Organ Transplant Recipients-Psoriasis is a chronic, immune-mediated, inflammatory disease with a prevalence of approximately 3\% in the United States. ${ }^{8}$ Approximately one-third of patients with psoriasis develop psoriatic arthritis. ${ }^{9}$ Organ transplant recipients with psoriasis and psoriatic arthritis represent a unique patient population whereby their use of chronic immunosuppressive medications to prevent graft rejection may put them at risk for developing infections and malignancies.

Special Considerations for Brodalumab-Brodalumab is an immunomodulatory biologic that binds to and inhibits IL-17RA, thereby inhibiting the actions of IL-17A, F, E, and C. ${ }^{2}$ The blockade of IL-17RA by brodalumab has been shown to result in reversal of psoriatic phenotype and gene expression patterns. ${ }^{10}$ Brodalumab was chosen as the treatment in our patient because it has a rapid onset of action, sustained efficacy, and an acceptable safety profile. ${ }^{11}$ Brodalumab is well tolerated, with approximately $60 \%$ of patients achieving clearance longterm. ${ }^{12}$ Candidal infections can occur in patients with brodalumab, but the rates are low and they are reversible with antifungal treatment. ${ }^{13}$ The increased mucocutaneous candidal infections are consistent with medications whose mechanism of action is IL-17 inhibition..$^{14,15}$ The most common adverse reactions found were nasopharyngitis and headache. ${ }^{16}$ The causal link between brodalumab and suicidality has not been established. ${ }^{17}$

The use of brodalumab for psoriasis in organ transplant recipients has not been previously reported in the literature. A few case reports have been published on the successful use of etanercept and ixekizumab as biologic treatment options for psoriasis in transplant patients. ${ }^{18-23}$ In addition to choosing an appropriate biologic for psoriasis in transplant patients, transplant providers may evaluate the choice of immunosuppression regimen for the organ transplant in the context of psoriasis. In a retrospective analysis of liver transplant patients with psoriasis, Foroncewicz et $\mathrm{al}^{3}$ found cyclosporine, which was used as an antirejection immunosuppressive agent in the posttransplant period, to be more effective than tacrolimus in treating recurrent psoriasis in liver transplant recipients.

Our case illustrates one example of the successful use of brodalumab in a patient with a solid organ transplant. Our patient's psoriasis and symptoms of psoriatic arthritis greatly improved after initiation of brodalumab. In the posttransplant period, the patient did not develop graft-vs-host disease, infections, malignancies, depression, or suicidal ideation while taking brodalumab.

\section{Conclusion}

It is important that the patient, dermatology team, and transplant team work together to navigate the challenges and relatively unknown landscape of psoriasis treatment in organ transplant recipients. As the number of organ transplant recipients continues to increase, this issue will become more clinically relevant. Case reports and future prospective studies will continue to inform us regarding the role of biologics in psoriasis treatment posttransplantation.

\section{REFERENCES}

1. Menter A, Strober BE, Kaplan DH, et al. Joint AAD-NPF guidelines of care for the management and treatment of psoriasis with biologics. J Am Acad Dermatol. 2019;80:1029-1072. 
2. Prussick R, Wu JJ, Armstrong AW, et al. Psoriasis in solid organ transplant patients: best practice recommendations from The Medical Board of the National Psoriasis Foundation. J Dermatol Treat. 2018;29:329-333.

3. Foroncewicz B, Mucha K, Lerut J, et al. Cyclosporine is superior to tacrolimus in liver transplant recipients with recurrent psoriasis. Ann Transplant. 2014;19:427-433.

4. Boukoum H, Nahdi I, Sahtout W, et al. BK and JC virus infections in healthy patients compared to kidney transplant recipients in Tunisia. Microbial Pathogenesis. 2016;97:204-208.

5. Bouwes Bavinck JN, Euvrard S, Naldi L, et al. Keratotic skin lesions and other risk factors are associated with skin cancer in organtransplant recipients: a case-control study in The Netherlands, United Kingdom, Germany, France, and Italy. J Invest Dermatol. 2007;127:1647-1656.

6. Clark CA, Robbins HA, Tatalovich Z, et al. Risk of Merkel cell carcinoma after transplant. Clin Oncol. 2019;31:779-788.

7. Lakhani NA, Saraiya M, Thompson TD, et al. Total body skin examination for skin cancer screening among U.S. adults from 2000 to 2010 Prev Med. 2014;61:75-80.

8. Rachakonda TD, Schupp CW, Armstrong AW. Psoriasis prevalence among adults in the United States. J Am Acad Dermatol. 2014; 70:512-516.

9. Alinaghi F, Calov M, Kristensen LE, et al. Prevalence of psoriatic arthritis in patients with psoriasis: a systematic review and metaanalysis of observational and clinical studies. J Am Acad Dermatol. 2019;80:251-265.

10. Russell CB, Rand H, Bigler J, et al. Gene expression profiles normalized in psoriatic skin by treatment with brodalumab, a human anti-IL-17 receptor monoclonal antibody. J Immunol. 2014;192:3828-3836.

11. Foulkes AC, Warren RB. Brodalumab in psoriasis: evidence to date and clinical potential. Drugs Context. 2019;8:212570. doi:10.7573 /dic. 212570

12. Puig L, Lebwohl M, Bachelez $\mathrm{H}$, et al. Long-term efficacy and safety of brodalumab in the treatment of psoriasis: 120 -week results from the randomized, double-blind, placebo- and active comparator-controlled phase 3 AMAGINE-2 trial. J Am Acad Dermatol. 2020;82:352-359.

13. Lebwohl M, Strober B, Menter A, et al. Phase 3 studies comparing brodalumab and ustekinumab in psoriasis. N Engl J Med. 2015; 373:1318-1328

14. Conti HR, Shen F, Nayyar N, et al. Th17 cells and IL-17 receptor signaling are essential for mucosal host defense against oral candidiasis. J Exp Med. 2009;206:299-311.

15. Puel A, Cypowyj S, Bustamante J, et al. Chronic mucocutaneous candidiasis in humans with inborn errors of interleukin-17 immunity. Science. 2011;332:65-68.

16. Farahnik B, Beroukhim B, Abrouk M, et al. Brodalumab for the treatment of psoriasis: a review of Phase III trials. Dermatol Ther. 2016;6:111-124.

17. Lebwohl MG, Papp KA, Marangell LB, et al. Psychiatric adverse events during treatment with brodalumab: analysis of psoriasis clinical trials. J Am Acad Dermatol. 2018;78:81-89.

18. DeSimone C, Perino F, Caldarola G, et al. Treatment of psoriasis with etanercept in immunocompromised patients: two case reports. J Int Med Res. 2016;44:67-71.

19. Madankumar R, Teperman LW, Stein JA. Use of etanercept for psoriasis in a liver transplant recipient. JAAD Case Rep. 2015; 1:S36-S37.

20. Collazo MH, González JR, Torres EA. Etanercept therapy for psoriasis in a patient with concomitant hepatitis $C$ and liver transplant. $P$ R Health Sci J. 2008;27:346-347.

21. Hoover WD. Etanercept therapy for severe plaque psoriasis in a patient who underwent a liver transplant. Cutis. 2007;80:211-214.

22. Brokalaki EI, Voshege N, Witzke O, et al. Treatment of severe psoriasis with etanercept in a pancreas-kidney transplant recipient. Transplant Proc. 2012;44:2776-2777.

23. Lora V, Graceffa D, De Felice C, et al. Treatment of severe psoriasis with ixekizumab in a liver transplant recipient with concomitant hepatitis B virus infection. Dermatol Ther. 2019;32:E12909. 